\title{
Aligned gap multiple split ring resonator biosensor for DNA hybridization detection at microwave frequency
}

\begin{abstract}
In this paper, the interest of microwave signals for biological detection applications using metamaterial structure as a biosensor element has been demonstrated. Microwave sensing is highly challenging for biological detection as it presents the attractive advantages of being contactless, non-invasive at low power level and yet its label free. The resonant frequency of the sensor is measured as $4.96 \mathrm{GHz}$. The shift in resonant frequency is employed as the sensor output for deoxyribonucleic acid (DNA) detection. The resonance frequency is shifted to the left when single stranded deoxyribonucleic acid (ssDNA) is immobilized onto gold surface and further shifted when hybridization or the binding event between the molecule of ssDNA and cDNA occurred. The sensitivity of this biosensor is up to $10 \mathrm{MHz} / \mathrm{Molar}$ with the detection limit of Molar is conducted during this experiment.
\end{abstract}

Keyword: Microwave biosensor; Metamaterial; Split ring resonator; Hybridization; DNA sensing 